\title{
New bounds on light sneutrino masses: Rare SUSY signals
}

\author{
Linda M. Carpenter, ${ }^{1, *}$ Humberto Gilmer, ${ }^{1, \dagger}$ and Junichiro Kawamura ${ }^{1,2, \$}$ \\ ${ }^{1}$ Department of Physics, The Ohio State University, Columbus, Ohio 43210, USA \\ ${ }^{2}$ Department of Physics, Keio University, Yokohama 223-8522, Japan
}

(Received 3 August 2020; accepted 8 April 2021; published 17 May 2021)

\begin{abstract}
We study bounds on a neutral component of a weak doublet scalar lepton. A typical example of such a particle is sneutrinos in supersymmetric models. Using constraints from invisible Higgs decays, we place a lower bound of approximately $\frac{m_{h}}{2}$. We recast a mono- $W / Z$ search with a hadronic vector-boson tag in order to bound parameter space in the sneutrino-charged slepton mass plane. We find a lower bound on sneutrinos in the range of $55-100 \mathrm{GeV}$ in the $36 \mathrm{fb}^{-1}$ data set depending on the mass of the charged component. We propose a sensitivity search in the hadronic mono- $W / Z$ channel for HL-LHC and discuss both the discovery potential in case an excess is seen and the exclusion limit assuming no excess is seen.
\end{abstract}

DOI: 10.1103/PhysRevD.103.095014

\section{INTRODUCTION}

Supersymmetry (SUSY) remains the leading candidate for beyond the Standard Model (BSM) physics. Lower bounds on colored superpartners are in the $\mathrm{TeV}$ range in standard decay scenarios. For example, lower bounds on gluinos are in the $2 \mathrm{TeV}$ range [1-4]. However, lower bounds on electroweak superpartners are not nearly as stringent, and many unexplored gaps in parameter space exist. Lower bounds on electroweak states are much less stringent. For example, bounds on charginos-which are nearly mass degenerate with the lightest supersymmetric particle (LSP) - are quite unrestricted ${ }^{1}$ in the $100 \mathrm{GeV}$ range, and largely come from LEP-II [6,7]. Among the least constrained sparticles are sneutrinos. The current completely generic lower mass bounds on sneutrinos are under $\frac{m_{Z}}{2}$ and actually come from the measurement of the $Z$ invisible width at LEP-I $[7,8]$.

In addition, collider signatures for the slepton sector are extremely dependent on the sparticle spectrum. Though neutralinos are the canonical candidate for the LSP in many models, it is possible for sneutrinos to be the LSP $[9,10]$. This includes versions of general gauge mediation [11-13],

\footnotetext{
*arpenter.690@osu.edu

† gilmer.30@osu.edu

*kawamura.14@osu.edu

${ }^{1}$ If their masses are so degenerate that charginos decay in detectors, they can be probed by searches for disappearing tracks $[5]$.

Published by the American Physical Society under the terms of the Creative Commons Attribution 4.0 International license. Further distribution of this work must maintain attribution to the author(s) and the published article's title, journal citation, and DOI. Funded by SCOAP ${ }^{3}$.
}

Dirac gaugino models [14-18], models with Yukawa textures [19], models with nonuniversal Higgs masses [20-23], and others. In minimal supersymmetric standard model (MSSM) realizations of SUSY models with a sneutrino LSP or next-to LSP (NLSP), the charged lefthanded slepton mass lies closely above the sneutrino mass. This means that charged sleptons decay to sneutrinos with very soft decay products. This makes charged slepton detection in the sneutrino LSP scenario difficult, as searches in standard channels [24,25] do not apply. In these scenarios both sneutrinos and charged sleptons appear in the decay chain as missing energy. New search strategies must be developed to explore this type of spectrum.

In this work we propose new sources for generic lower mass bounds on light sneutrinos. We first place a modelindependent lower mass bound by investigating couplings in the Higgs sector. Through four scalar interactions, the Higgs boson may couple to sneutrinos; we then bound the light sneutrino parameter space with the Higgs width constraints. We then propose a new search strategy for the slepton sector for models with a sneutrino LSP/NLSP by employing mono-boson searches. Mono-particle searches have been very useful in looking for various BSM phenomena, including compressed SUSY particles [26-28] and dark matter (DM) searches [29,30]. In this work, we choose to recast LHC dark matter searches for heavy mono-electroweak bosons [31] to constrain models with light sneutrinos. In particular, we propose a recast of the $13 \mathrm{TeV}$ ATLAS hadronic mono- $W / Z$ search: $p p \rightarrow \tilde{\ell} \tilde{\ell}^{*}+W / Z(\rightarrow j j)$, where $\tilde{\ell}$ is a slepton which contains both sneutrinos $\tilde{\nu}$ and charged sleptons $\tilde{e}$. We then perform a sensitivity search in this channel for $3 a b^{-1}$ of the full $14 \mathrm{TeV}$ run at the HL-LHC. 
This paper is organized as follows. In Sec. II we review sneutrinos in the MSSM and define a simplified model with sneutrinos to be used for our phenomenological analysis. In Sec. III we study constraints on light sneutrinos. At first, we explore the parameter space bound on light sneutrinos from Higgs decays. We then discuss the mono- $W / Z$ hadronic search at the LHC. Current constraints and future sensitivities in the sneutrino-charged slepton mass plane are shown in Sec. III C. We conclude in Sec. IV.

\section{SNEUTRINO MODELS}

In this paper, we study the phenomenology of a light $S U(2)_{L}$ doublet scalar lepton (slepton) which may have a nonzero lepton number and is distinguished from Higgs doublets. A typical example is doublet (left-handed) sleptons in SUSY extensions of the SM. Extra sleptons are also introduced in models that explain DM and/or the discrepancy of $(g-2)_{\mu}$ from the SM prediction $[7,32]$.

\section{A. Sneutrino (N)LSP models in MSSM}

There are many implementations of SUSY models where sneutrinos are the lightest MSSM sparticle. Some of the models of sneutrino LSPs were detailed in Refs. [23,33]. The parameter space of general gauge mediation also contains many regions in which the sneutrino is the NLSP and may decay to a light gravitino $[12,13]$. Although we will not consider $R$-parity violation here, it has been noted that in models with $R$-parityviolating operators, the renormalization group (RG) effects make the sneutrino masses light [19,34]. The light sleptons might be helpful to explain the longstanding discrepancy in the anomalous magnetic moment of the muon, $(g-2)_{\mu}$ [35-38].

In this paper, we consider the case that the sneutrino is the lightest particle in the MSSM, but is not a sizable fraction of the DM. It is well known that the sneutrino LSP is excluded as the DM candidate due to the strong constraints from direct detection [39-41]. The Z-boson exchange induces the sizable DM-nucleus scattering cross section. This constraint can be evaded if the relic density of the sneutrino is so tiny that the detection rate is below the current bounds, although other DM candidates are required in this case. ${ }^{2}$ Another possibility is that the sneutrino is the NLSP and there is a lighter SUSY particle, such as a gravitino [45-49] or an axino [50-54].

In the MSSM, the relevant terms in the scalar potential for doublet sleptons $\tilde{\ell}_{i}=\left(\tilde{\nu}_{i}, \tilde{e}_{i}\right)$ and the neutral components in the Higgs doublets $h_{d}^{0}, h_{u}^{0}$ are given by

\footnotetext{
${ }^{2}$ Mass splitting between the $C P$-even and -odd states in the sneutrino will reduce the direct-detection rate and some parameter space would be viable [42-44].
}

$$
\begin{aligned}
V_{\tilde{e}}^{\mathrm{MSSM}}= & \sum_{i=1,2,3}\left[m_{L_{i}}^{2}\left(\left|\tilde{\nu}_{i}\right|^{2}+\left|\tilde{e}_{i}\right|^{2}\right)\right. \\
& +\frac{g^{2}}{4}\left(\left|\tilde{\nu}_{i}\right|^{2}-\left|\tilde{e}_{i}\right|^{2}\right)\left(\left|h_{d}^{0}\right|^{2}-\left|h_{u}^{0}\right|^{2}\right) \\
& \left.+\frac{g^{\prime 2}}{4}\left(\left|\tilde{\nu}_{i}\right|^{2}+\left|\tilde{e}_{i}\right|^{2}\right)\left(\left|h_{d}^{0}\right|^{2}-\left|h_{u}^{0}\right|^{2}\right)\right],
\end{aligned}
$$

where $g^{\prime}$ and $g$ are the gauge coupling constants of $U(1)_{Y}$ and $S U(2)_{L}$, respectively, and $m_{L_{i}}^{2}$ is the soft mass squared of the $i$ th doublet slepton. The quartic terms in the second line come from the $D$-term potential. Throughout this paper, we assume that the soft mass $m_{L_{i}}$ is flavor independent and singlet charged sleptons are much heavier than the doublet ones. Hereafter, we omit the flavor index $i$ for simplicity. From the latter assumption, the left-right mixing in the charged sleptons may be negligible due to suppression by the heavy singlet state as well as the small Yukawa couplings.

The left-handed charged sleptons are not split very much from the sneutrinos since the charged and neutral states are given by a single soft mass parameter $m_{L}^{2}$. In the absence of left-right mixing in the charged sleptons, the mass splitting between the states is induced only by the $D$ term of $S U(2)_{L}$ and is given by

$$
\Delta m:=m_{\tilde{e}}-m_{\tilde{\nu}}=-\frac{m_{W}^{2} \cos 2 \beta}{m_{\tilde{e}}+m_{\tilde{\nu}}},
$$

where $\tan \beta:=\left\langle h_{u}^{0}\right\rangle /\left\langle h_{d}^{0}\right\rangle$, and $m_{\tilde{e}}$ and $m_{\tilde{\nu}}$ are the charged slepton and sneutrino mass, respectively. Here, the mass splitting decreases with increasing slepton masses and is $\mathcal{O}(10) \mathrm{GeV}$ for $\mathcal{O}(100) \mathrm{GeV}$ states. This relation implies that the mass splitting is at most the $W$-boson mass in the MSSM. ${ }^{3}$

\section{B. Sneutrinos in general models}

The search strategies for sleptons discussed in the next section will be applicable for a more general class of scalar fields, consisting of a weak doublet with hypercharge $Y=\frac{1}{2}$ such that a neutral component sneutrino is present. This is because we exploit only the invisibility of sneutrinos in the SM boson decays and mono- $W / Z$ searches. Hence, our study will be applicable to, e.g., the inert Higgs doublet [55]. ${ }^{4}$ For example, scalar doublet leptons are

\footnotetext{
${ }^{3}$ The sizable left-right mixing in the charged slepton mass matrix will decrease the mass of the lighter state, and it will never increase the mass splitting from the sneutrino as long as the sneutrino is lighter than the charged ones. Hence, this bound will hold even if there is sizable left-right mixing.

${ }^{4}$ One big difference is that there is only one inert Higgs doublet in the minimal setup, while there are three generations of sneutrinos in the MSSM. Even if sneutrinos decay to a lighter particle the limits from our study will be particularly applicable for a mass-degenerate region.
} 
introduced to explain the long-standing discrepancy of $(g-2)_{\mu}$ and DM [56-60]. It is shown that the DM and slepton masses may need to be degenerate, so that the direct-detection rate is sufficiently suppressed, while the relic density is explained by the thermal freeze-out mechanism with the help of coannihilation. The mono- $Z / W$ search may be a unique way to probe such a massdegenerate region at the LHC. In non-SUSY models, quartic couplings to Higgs bosons are not related to the gauge couplings. This may allow a mass difference larger than the bound in Eq. (2), although too large quartic couplings may induce lower Landau pole scales.

Even larger mass splitting may be induced by new $S U(2)_{L}$ triplet states which have trilinear couplings with sleptons,

$$
-\mathcal{L}_{T}:=A_{T} \tilde{\ell}^{\dagger} T \tilde{\ell}+\text { H.c., }
$$

where $T$ is a triplet scalar and $A_{T}$ is a trilinear coupling constant. The mass-squared difference in the sleptons is given by $A_{T}\left\langle T^{0}\right\rangle$, so that the mass difference can be, in principle, much larger than the $W$-boson mass by $A_{T} \gg m_{W}$. These types of triplet couplings are induced in the SUSY models with Dirac gauginos [61]. More general models may include weak triplets with nonzero vacuum expectation values, e.g., in the seesaw mechanisms for light neutrino masses [62-67]. Note that triplets can easily change the electroweak precision observables from the SM values [7]. This will considerably restrict parameter spaces of models with triplets.

In this work we take a phenomenological, simplified model approach to the light slepton parameter space. The slepton potential in the simplified model is defined as

$$
V_{\tilde{e}}=m_{\tilde{\nu}}^{2}|\tilde{\nu}|^{2}+m_{\tilde{e}}^{2}|\tilde{e}|^{2}+A_{\tilde{\nu}} h|\tilde{\nu}|^{2}+\frac{1}{2} \lambda_{\tilde{\nu}} h^{2}|\tilde{\nu}|^{2},
$$

where $h$ is the physical $C P$-even scalar of the SM Higgs boson. In the MSSM, the effective trilinear and quartic coupling constants $A_{\tilde{\nu}}$ and $\lambda_{\tilde{\nu}}$ are given by

$$
A_{\tilde{\nu}}=\frac{g m_{Z}^{2}}{2 m_{W}} \sin (\alpha+\beta), \quad \lambda_{\tilde{\nu}}=\frac{g^{2} m_{Z}^{2}}{4 m_{W}^{2}} \cos 2 \alpha,
$$

where the neutral complex Higgs bosons are expanded as

$$
\begin{aligned}
\left(\begin{array}{l}
h_{u}^{0} \\
h_{d}^{0}
\end{array}\right)= & \left(\begin{array}{c}
\left\langle h_{u}^{0}\right\rangle \\
\left\langle h_{d}^{0}\right\rangle
\end{array}\right)+\frac{1}{\sqrt{2}}\left(\begin{array}{cc}
\cos \alpha & \sin \alpha \\
-\sin \alpha & \cos \alpha
\end{array}\right)\left(\begin{array}{l}
h \\
H
\end{array}\right) \\
& +\frac{i}{\sqrt{2}}\left(\begin{array}{cc}
\sin \beta_{0} & \cos \beta_{0} \\
-\cos \beta_{0} & \sin \beta_{0}
\end{array}\right)\left(\begin{array}{l}
G \\
A
\end{array}\right) .
\end{aligned}
$$

Here, $H(A)$ is a $C P$-even (-odd) Higgs boson and $G$ is a Nambu-Goldstone boson mode which is absorbed by the $Z$ boson. We note that the quartic coupling with the Higgs boson will not play a significant role in the mono- $W / Z$ search studied in the next section, since the production cross sections involving the Higgs bosons are negligible compared to those involving the $W / Z$ bosons. We have checked that this is true in the MSSM, and hence we will not consider the effect of the quartic coupling in the following analysis.

\section{PHENOMENOLOGY}

We discuss constraints on slepton masses in a simplified model defined in Eq. (4) from invisible Higgs boson decays and LHC searches. In this paper, we assume the following. ${ }^{5}$

1. Sneutrinos and charged sleptons have universal masses and couplings, respectively.

2. Sneutrinos are stable, or decay to invisible particles.

3. Charged sleptons exclusively decay to the sneutrinos through the gauge coupling.

In the MSSM, these will be realized when the doublet sleptons are the lightest MSSM particle and the effects of the tau Yukawa coupling are negligible. If the tau Yukawa coupling is sizable due to, e.g., large $\tan \beta$, the tau slepton might be lighter than the others through RG effects. Limits obtained under the universal assumption will give conservative limits for the lighter stau scenarios.

\section{A. Higgs invisible decays}

The current model-independent limit on the sneutrino mass is obtained from invisible decay of the $Z$ boson [7]. ${ }^{6}$ We point out that invisible decay of the Higgs boson constrains the sneutrino mass in a model-independent way. The limit is tightened because of the heavier mass of the Higgs boson than the $Z$ boson. Note that no gauge symmetry can forbid the quartic coupling $\lambda|\tilde{\ell}|^{2}|H|^{2}$, which induces the Higgs decay to a pair of sneutrinos through the effective $A$ term of $\mathcal{O}\left(\lambda v_{H}\right)$. In fact, the $A$ term in Eq. (5) is of this order in the MSSM.

The decay width to sneutrinos is given by

$$
\Gamma\left(h \rightarrow \tilde{\nu} \tilde{\nu}^{*}\right)=N_{\tilde{\nu}} \frac{\left|A_{\tilde{\nu}}\right|^{2}}{16 \pi m_{h}} \sqrt{1-\frac{4 m_{\tilde{\nu}}^{2}}{m_{h}^{2}}},
$$

where $N_{\tilde{\nu}}$ is the number of sneutrinos. The branching fraction of the invisible decay to sneutrinos is evaluated as

\footnotetext{
${ }^{5}$ As mentioned in Sec. II A, the sneutrino density should comprise a negligible percent of the total DM density if the sneutrino is stable enough to be DM.

${ }^{6}$ The limit of $m_{\tilde{\nu}}>94 \mathrm{GeV}[6,7]$ is obtained under an assumption of the constrained MSSM spectrum.
} 

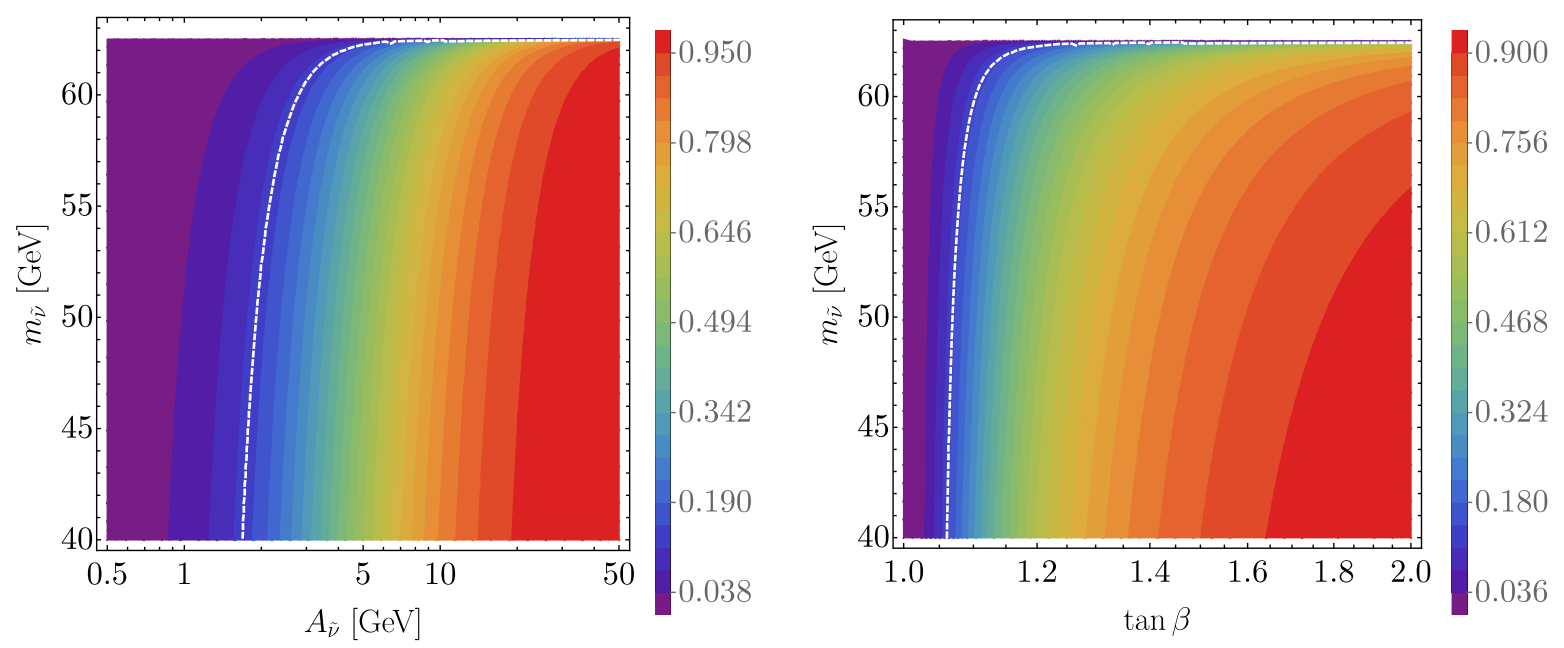

FIG. 1. Contours of Higgs to invisible branching fractions in the general model (left) and in the MSSM (right). The white lines show the current limit of $\operatorname{Br}(h \rightarrow$ inv $)=0.13$ [68].

$$
\begin{aligned}
\operatorname{Br}(h \rightarrow \text { inv }):= & \frac{\Gamma\left(h \rightarrow \tilde{\nu} \tilde{\nu}^{*}\right)}{\Gamma_{h}} \sim 390 \\
& \times\left(\frac{4.07[\mathrm{MeV}]}{\Gamma_{h}}\right)\left(\frac{\left|A_{\tilde{\nu}}\right|}{100[\mathrm{GeV}]}\right)^{2} \\
& \times N_{\tilde{\nu}} \sqrt{1-\frac{4 m_{\tilde{\nu}}^{2}}{m_{h}^{2}}}
\end{aligned}
$$

where $\Gamma_{h}$ is the total decay rate of the SM Higgs boson. Hence, the invisible decay tends to dominate over the Higgs decay if it is kinematically allowed, ${ }^{7}$ while the current limit on the invisible decay branching fraction is 0.13 [68]. Thus, a sneutrino lighter than half of the Higgs mass is excluded unless the effective $A$ term is suppressed as $\mathcal{O}(1 \mathrm{GeV})$ by the small quartic couplings and/or Higgs mixing angle.

In the MSSM with the decoupling limit of the heavy Higgs bosons, $\alpha \sim \beta-\pi / 2$,

$$
\sin (\alpha+\beta) \sim-1+\frac{2}{1+\tan ^{2} \beta} .
$$

Thus, the invisible decay rate is suppressed by the mixing angle when $\tan \beta \sim 1$; otherwise, $m_{\tilde{\nu}}<\frac{m_{h}}{2}$ is immediately excluded. Figure 1 shows the region excluded by the invisible decay of the SM Higgs boson in the general model (left) and in the MSSM (right). For the MSSM plot, we impose the decoupling limit value $\alpha=\beta-\pi / 2$. When $m_{\tilde{\nu}} \lesssim m_{h} / 2$, the upper bound on the effective $A$ term is about $2 \mathrm{GeV}$ and this bound corresponds to $\tan \beta \lesssim 1.1$ in the MSSM. Potential problems in the case of such

\footnotetext{
${ }^{7}$ The total decay rate itself is also constrained, $\Gamma_{h}<$ $0.013 \mathrm{GeV}$ [7], but this is much weaker than that from the branching fraction of invisible decay, since the bound of $0.013 \mathrm{GeV}$ is much larger than the total width of the SM Higgs boson, $4.07 \times 10^{-3} \mathrm{GeV}$.
}

small $\tan \beta \sim 1$ are possibly large values for the top Yukawa coupling constant and a small tree-level SM-like Higgs mass. The top Yukawa coupling constant will blow up below the conventional grand unification scale, $\sim 10^{16} \mathrm{GeV}$, depending on the precise value of the top Yukawa and gauge coupling constants. The existence of the Landau pole would make it difficult to interpret the MSSM as the low-energy theory of conventional grand unification theories. The other problem is that the SUSY-breaking masses, particularly top-squark masses, need to be heavier than $10^{8} \mathrm{GeV}$ to explain the $125 \mathrm{GeV}$ Higgs boson mass due to the small tree-level contribution [69]. Hence, it would be difficult to justify the lightness of sneutrinos regardless of the heavy squarks. If we consider these problems serious, it may be necessary to consider $\tan \beta \gtrsim 1.2$, and hence the sneutrinos lighter than half the Higgs boson mass are immediately excluded.

\section{B. LHC constraints}

\section{Mass spectra and signals of charged slepton}

In the light sneutrino model, the charged slepton will decay to a sneutrino through a $W$ boson, where this $W$ boson will either be on shell or off shell depending on the mass splitting. We can then divide the mass parameter space into three regions by the mass difference, $\Delta m:=m_{\tilde{e}}-m_{\tilde{\nu}}$,
1) $\Delta m \lesssim 20 \mathrm{GeV}$,
2) $20 \mathrm{GeV} \lesssim \Delta m<m_{W}$,
3) $m_{W}<\Delta m$.

We visualize these regions in Fig. 2. In the first region the mass compression is such that any decay products of the off-shell $W$ boson resultant from the charged slepton decay will be very soft, and thus charged sleptons produced in 


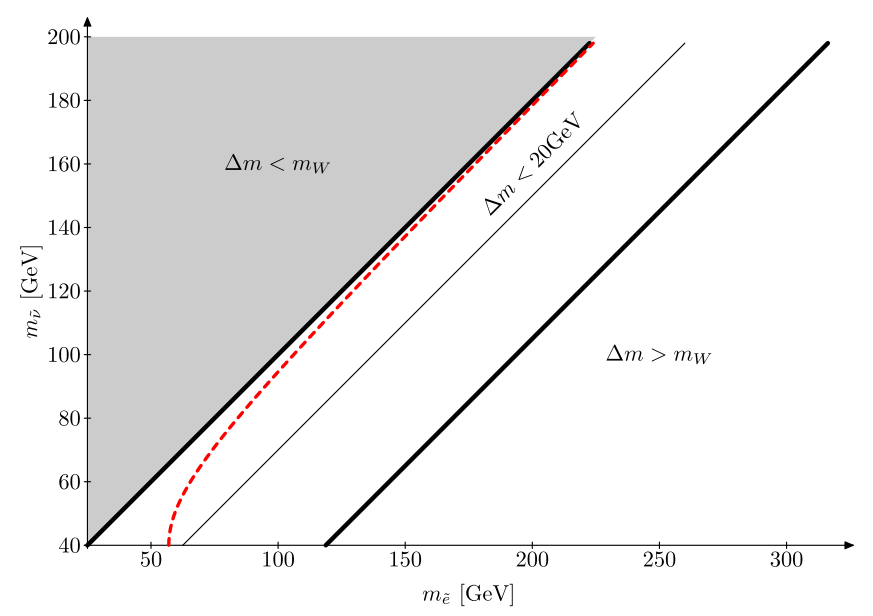

FIG. 2. Sneutrino-slepton parameter space, showing the various regions explored. The region of mass splitting above the red line is consistent with the MSSM for reasonable values of $\tan \beta$.

electroweak processes will likely appear in searches as missing energy. In the second region, the jets or leptons resultant from the $W$ decay will be hard enough to observe but would not be enough to reconstruct a $W$-boson-like object. In the third region, the slepton will decay to a sneutrino via an on-shell $W$, which will be reconstructed as a $W$-boson-like object.

Although we consider the parameter space where the charged sleptons are heavier than the sneutrinos, there might be constraints from the LHC searches for the pair production of charged sleptons,

$$
p p \rightarrow \tilde{e} \tilde{e} \rightarrow W^{(*)} W^{(*)} \tilde{\nu} \tilde{\nu}
$$

where the $W$ bosons are off shell in the first and second regions and are on shell in the third region. ${ }^{8}$ This signal is the same as that from the pair production of charginos [24].

Figure 3 shows the signal cross sections of doublet charged sleptons (red solid) and wino-like charginos (yellow dashed) at $\sqrt{s}=13 \mathrm{TeV}$. The production cross sections were calculated in Refs. [70-74] and in Refs. [71,75], respectively. Here, the slepton production cross section is calculated for the three-flavor sneutrinos. The blue thick and gray dashed lines are the exclusion limits on the signal cross sections where the neutral particle is 50 and $100 \mathrm{GeV}$, respectively. The values are shown in the supplemental material of Ref. [24]. The efficiency times acceptance factor $(\epsilon \times \mathcal{A})$ for the signal cross section is set to $10^{-3}$ such that a chargino within about $[200,400] \mathrm{GeV}$ is excluded by the exclusion limit for $m_{\tilde{\chi}^{0}}=100 \mathrm{GeV}$. Assuming the same efficiency times acceptance factor, we see that the charged slepton pair production will not be

\footnotetext{
${ }^{8}$ Hereafter, we will omit symbols for the conjugation of sleptons*, but we use this symbol for off-shell particles.
}

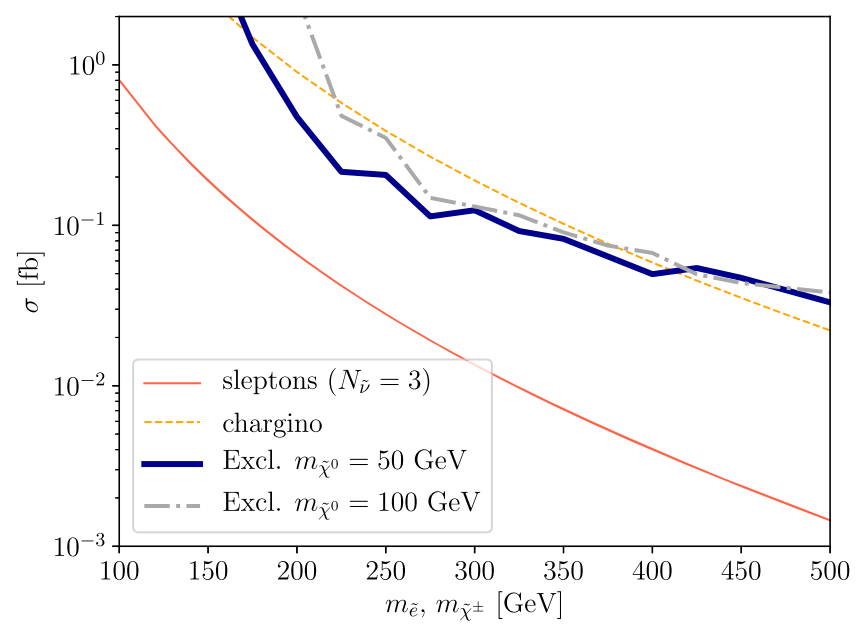

FIG. 3. Comparison of the signal cross section of a charged slepton pair with experimental limits from the chargino search [24].

detectable at the LHC due to the too small production cross section.

\section{Sneutrino signals}

As light sneutrinos appear as missing energy in the detector, events with final-state sneutrinos should be produced in association with a visible particle. As such, we choose to focus on LHC searches where sneutrinos are produced in association with a single boson. The monojet analysis, whose events result from an initial-state radiation (ISR) gluon, clearly has the largest production cross section; however, since there is a very large background the search will likely not have a great sensitivity to the sneutrino parameter space. For example, projections for the $3 \mathrm{ab}^{-1}$ run of the LHC using the monojet search can bound electroweak gauginos at about $100 \mathrm{GeV}$ [27], and slepton production cross sections are much smaller. The likely signal-to-background ratio does not bode well for this channel.

We thus turn to the production of sneutrinos and charged sleptons in association with the electroweak bosons. In Fig. 4, we plot cross sections versus sneutrino masses for sneutrinos pair produced in association with various electroweak bosons: the $W, Z$, photon, and Higgs boson. In this plot, the charged sleptons are fixed at $200 \mathrm{GeV}$. Note that in the mono-Higgs case, the Higgs boson is produced as final-state radiation (FSR) off of an internal gauge boson line, or off of a sneutrino line due to the four-scalar interaction mentioned previously. The photon is produced as initial-state radiation. $W$ production, however, may originate from ISR, FSR, or from an on-shell particle decay. We see that the $W$-boson production dominates the production cross sections. Balancing between production cross sections and anticipated signal-to-background ratios, we have chosen to recast the ATLAS mono-boson analysis 


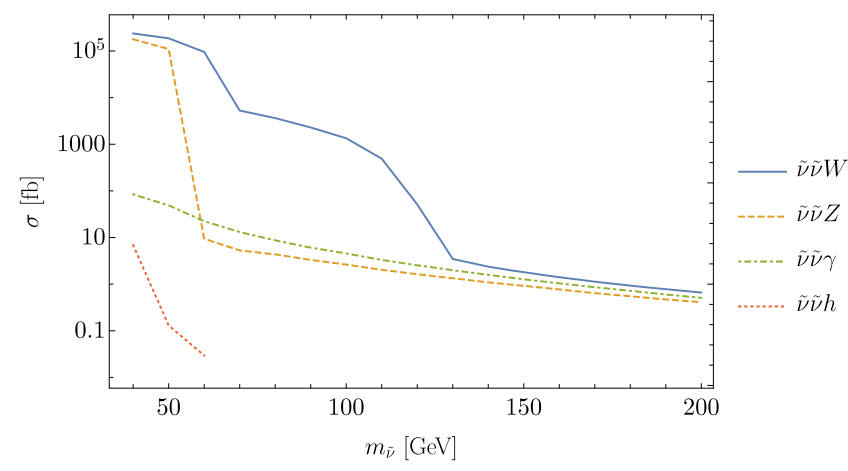

FIG. 4. Production cross sections for a sneutrino pair in association with the electroweak bosons. The charged slepton mass is fixed at $m_{\tilde{e}}=200 \mathrm{GeV}$.

[31] which searches for an on-shell hadronically tagged $W$ or $Z$ boson produced in association with large missing energy, $p p \rightarrow E_{T}^{\text {miss }}+W / Z(\rightarrow j j)$.

We now discuss how this search applies to our three regions of parameter space in the sneutrino-charged slepton mass plane:

1) In the mass-degenerate region both the sneutrinos and the softly decaying charged sleptons will appear as missing energy in the search. This means that not only does sneutrino pair production contribute to the overall production cross section, but so does the production of a sneutrino-charged slepton pair, and the pair production of softly decaying charged sleptons. The relevant processes are $p p \rightarrow W / Z+\tilde{\ell} \tilde{\ell}$, where $\tilde{\ell}$ contains both $\tilde{\nu}$ and $\tilde{e}$.

2) In the intermediate region, decays of the charged slepton are hard enough for decay products to be detected; however, since the decay products of the resultant $W$ boson are off shell, the events will not have $W / Z$-tagged jets. In this region, the only contributing process will be the pair production of

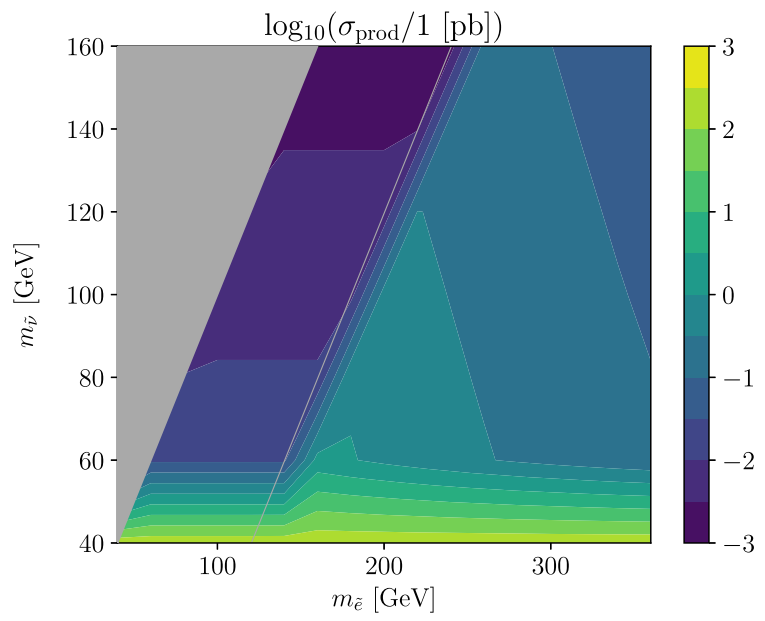

sneutrinos in association with a $W$ or $Z$ boson: $p p \rightarrow W / Z+\tilde{\nu} \tilde{\nu}$.

$3)$ In the large-mass-splitting region, two types of events contribute to the overall cross section. One is of course the pair production of sneutrinos in association with a $W$ boson as above, $p p \rightarrow W / Z+$ $\tilde{\nu} \tilde{\nu}$. However, the main production cross section results from the production of one sneutrino and one charged slepton. The charged slepton then undergoes a decay to a sneutrino through an on-shell $W$, which will be caught in the hadronic vector-boson tag. The relevant process is $p p \rightarrow \tilde{e} \tilde{\nu} \rightarrow W \tilde{\nu} \tilde{\nu}$.

In considering the large-mass-splitting region we may further understand the features of Fig. 4. In the $W$ and $Z$ associated channels we see that the production drops after the sneutrino mass passes $m_{Z} / 2$ as there is an enhancement for light sneutrinos produced by the decay of an on-shell $Z$ boson. There is an additional feature in the mono- $W$ channel. With the charged slepton mass fixed at $200 \mathrm{GeV}$, we see that for sneutrino masses lighter than about $120 \mathrm{GeV}$, the slepton mass splitting is large and the $W \tilde{\nu} \tilde{\nu}$ cross section is dominated by the production of a charged slepton-sneutrino pair. The slepton decays to the on-shell $W$ and a sneutrino. This production cross section is quite high. Once the sneutrino mass becomes large enough, the on-shell $W$ cannot be produced by the slepton decay. Instead, the on-shell vector boson must be produced as ISR/ FSR. This production has an implicitly lower cross section.

Figure 5 shows the total production cross sections $\tilde{\nu} \tilde{\nu}+W / Z$ in the sneutrino-charged slepton mass plane. The production cross section is dominated by $\tilde{\nu} \tilde{\nu}$ production in association with initial-/final-state $W / Z$ bosons where $m_{\tilde{\nu}} \lesssim 60 \mathrm{GeV}$. For heavier sneutrinos, the production cross section is smaller than $\mathcal{O}(0.01)$ pb in the off-shell $W$ region, while it can be $\mathcal{O}(0.1) \mathrm{pb}$ in the on-shell $W$ region due to the $\tilde{e} \tilde{v}$ production. The cross section slowly decreases as the charged slepton mass increases.

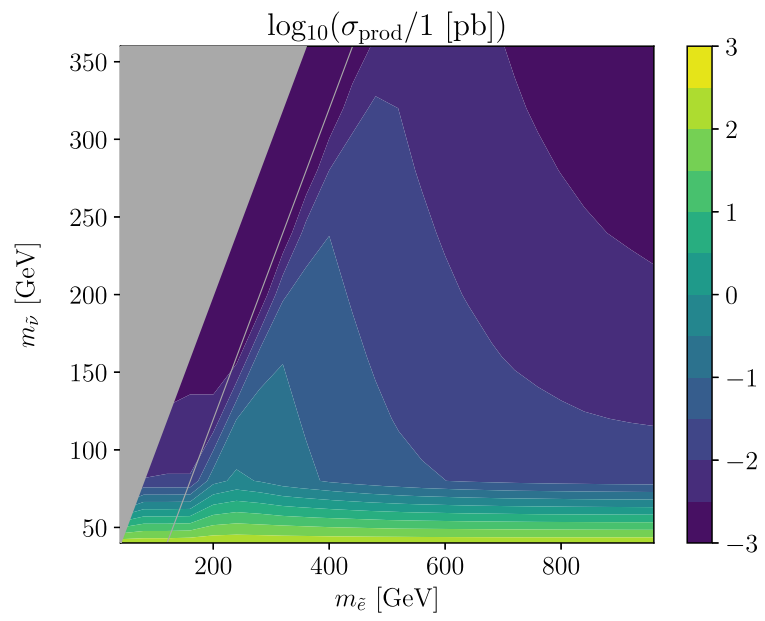

FIG. 5. Contour plot of production cross sections of $p p \rightarrow W / Z+\tilde{\nu} \tilde{\nu}$ at $\sqrt{s}=13 \mathrm{TeV}$ (left) and $14 \mathrm{TeV}$ (right) in the ( $\left.m_{\tilde{e}}, m_{\tilde{\nu}}\right)$ plane. 
The charged slepton mass dependence of the cross section is milder than that of the pair production. In the next section, we describe our recast of the ATLAS hadronictagged mono- $W / Z$ search and set limits in the sneutrinocharged slepton mass plane.

\section{Mono-W/Z search}

ATLAS has searched for a single on-shell hadronically decaying vector boson produced in association with invisible particles [31]. This search was originally conceived as a search for fermionic models of DM; however, we may recast this search in order to constrain the light sneutrino scenario.

The event selection criteria in the mono- $W / Z$ search are summarized in Table I. The search looks for events with large missing energy that contain a large- $R$ jet (merged topology) or two jets (resolved topology) with a dijet invariant mass falling within a window around the $W / Z$ boson mass. In both topologies, large missing transverse energy $\left(E_{T}^{\text {miss }}\right)$ is required and any events with reconstructed leptons are rejected. In order to suppress multijet backgrounds, azimuthal separation between the $E_{T}^{\text {miss }}$ vector and the large- $R$ jet (the two highest- $p_{T}$ jets system) is required to be larger than $2 \pi / 3$ in the merged (resolved) topology. The azimuthal separations between the $E_{T}^{\text {miss }}$ vector and the
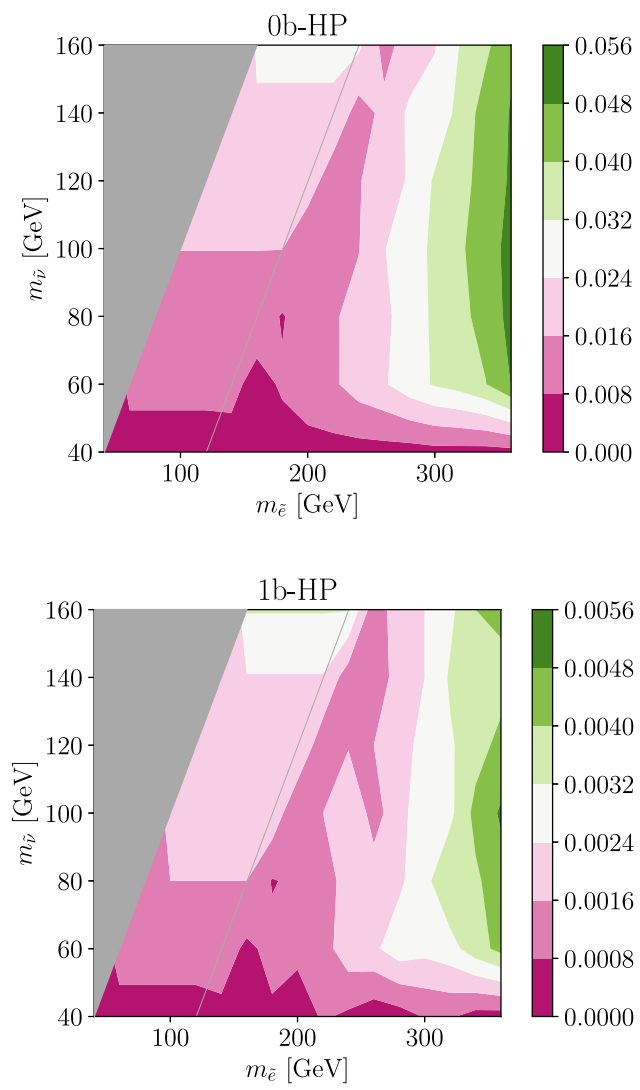

leading three jets are also required. In addition, track-based missing transverse momentum $\vec{p}_{T}^{\text {miss }}$, defined as the negative vector sum of the transverse momenta of tracks with $p_{T}>0.5 \mathrm{GeV}$ and $|\eta|<2.5$, is required to be larger than $30 \mathrm{GeV}$ and its azimuthal angle is close to that of the calorimeter-based $E_{T}^{\text {miss }}$ within $\pi / 2$.

In the merged topology, any $b$-tagged jets outside of the large- $R$ jet are rejected on top of the above requirements. The signal regions in this topology are separated by the number of $b$-tagged jets and the purity of the large- $R$ jet to be tagged as originating from a hadronic vector-boson decay. Since the selection criteria in the analysis [31] is adjusted such that the efficiency is 50\% independent of jet $p_{T}$, we simply assume that half of the events with a large- $R$ jet are classified into the high-purity (HP) regions, and the rest of the events are classified into the low-purity (LP) regions. Since the sneutrino pair production is mostly associated with a $W$ boson, there are very few events with two $b$-tagged jets. In fact, we found that the number of events in the signal region with two $b$-tagged jets is always less than the experimental limit. Hence, we study the signal regions with up to one $b$-tagged jet. Thus, we study the four signal regions $0 \mathrm{~b}-\mathrm{HP}, 0 \mathrm{~b}-\mathrm{LP}, 1 \mathrm{~b}-\mathrm{HP}$, and $1 \mathrm{~b}-\mathrm{LP}$.

In the resolved topology, the highest- $p_{T}$ jet is required to be $p_{T}>45 \mathrm{GeV}$ and the sum of the $p_{T}$ 's of the two (three)
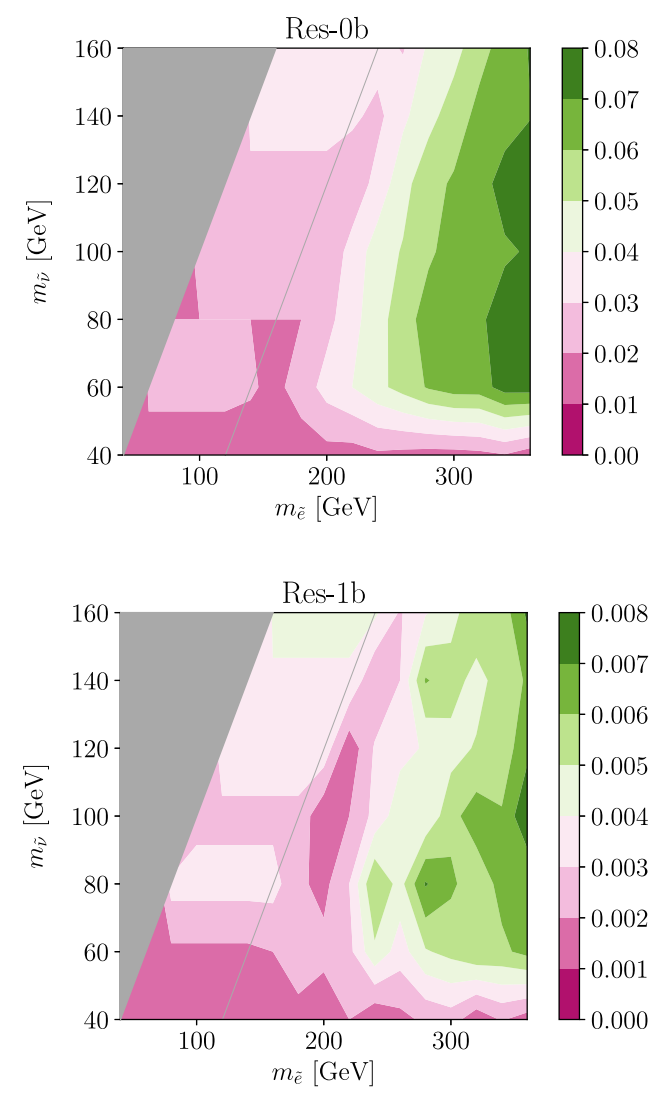

FIG. 6. Efficiencies of sneutrino production in association with a $W / Z$ boson in the $0 \mathrm{~b}-\mathrm{HP}$ (top left), Res-0b (top right), 1b-HP (bottom left), and Res-1b (bottom right) regions at $\sqrt{s}=13 \mathrm{TeV}$. 
leading jets is required to be larger than 120 (150) GeV. In addition, the angular separation $\Delta R:=\sqrt{(\Delta \phi)^{2}+(\Delta \eta)^{2}}$ between the two leading jets and the invariant mass of the two leading jets are required to be smaller than 1.4 and within the range $[65,105] \mathrm{GeV}$, respectively. Events in this topology are classified into three signal regions by the number of $b$-tagged jets. We study the two signal regions 0b-Res and $1 b$-Res.

We have generated signal events using MadGraph5 2.7.2 [76], showered events with Pythia 8.2.4.5 [77], and ran events through the fast detector simulator Delphes3 [78]. We used the default ATLAS card for the detector simulation, but we added the large- $R$ jet with $R=1.0$ on top of the small- $R$ jet with $R=0.4$ reconstructed by using the anti- $k_{T}$ jet clustering algorithm [79,80]. The trimming algorithm [81] is applied to the large- $R$ jets as in the analysis of Ref. [31], where subjets with $R=0.2$ are removed from the large- $R$ jet if their transverse momentum is less than $5 \%$ of the original large- $R$ jet transverse momentum. We generated events with $\sqrt{s}=13 \mathrm{TeV}$ to recast the current experimental limit, and we use the same configuration to study future sensitivities at the HL-LHC but the center-of-mass energy is set to $\sqrt{s}=14 \mathrm{TeV}$.
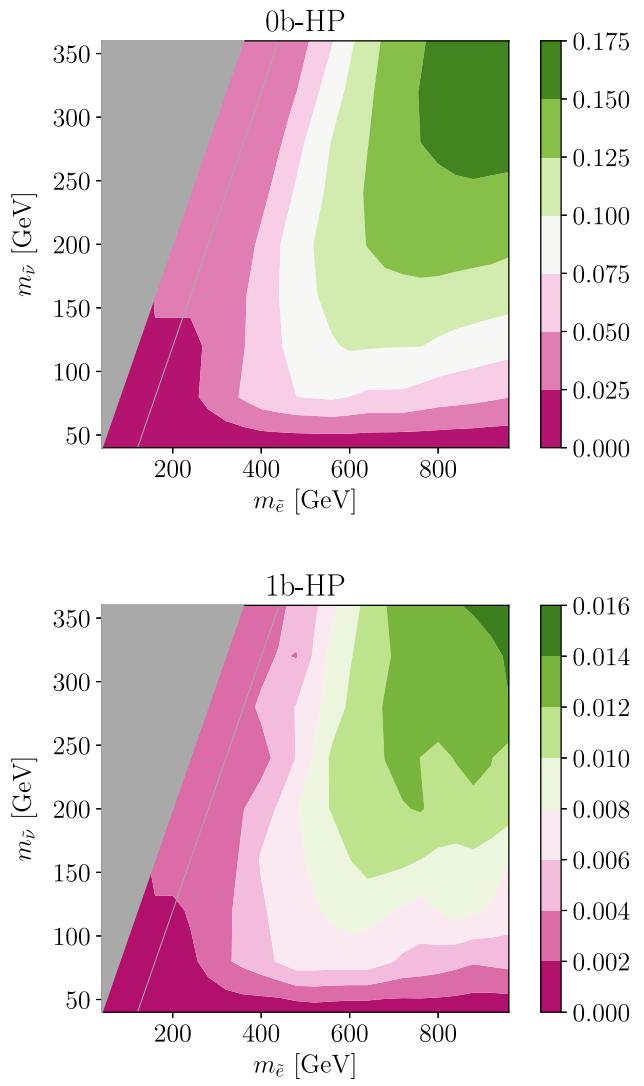

Figures 6 and 7 show the efficiencies of the sneutrino productions to the signal regions defined in Table I when $\sqrt{s}=13$ and $14 \mathrm{TeV}$, respectively. $m_{\tilde{\nu}}>m_{\tilde{e}}$ in the gray region. Since we assume that half of the events with a large$R$ jet are classified into the HP regions, the efficiency factors of the LP regions are the same as those of the HP regions.

Events from the production with an initial-/final-state $W / Z$ boson tend to have smaller efficiencies, while those with a $W$ boson from the $\tilde{e}$ decay tend to have larger efficiencies. The $p_{T}$ distribution of the latter is centered according to the mass difference between the charged slepton and sneutrino. In the merged topology signal regions, the efficiency increases as the mass difference increases. With fixed $m_{\tilde{\nu}}$, the efficiency decreases slightly as $m_{\tilde{e}}$ increases, because the production of $\tilde{e} \tilde{\nu}$ becomes less important against the production with an initial-/final-state $W / Z$ boson. The $W / Z$ boson should be off shell in order to be categorized using the resolved topology; this is so that the efficiency is maximized where the mass splitting is moderately large, on the order of $\sim 300 \mathrm{GeV}$. No bottom quark is produced from $W$ boson decays. The efficiencies to the signal regions with $b$-tagged jets are significantly
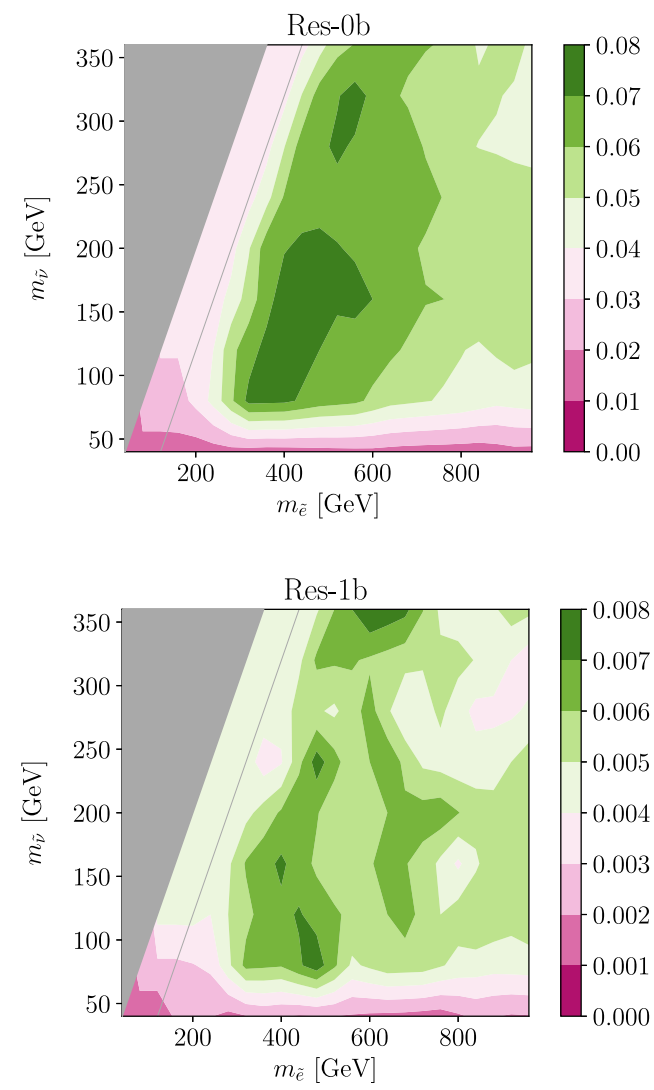

FIG. 7. Efficiencies of sneutrino production in association with a $W / Z$ boson in the 0b-HP (top left), Res-0b (top right), $1 \mathrm{~b}-\mathrm{HP}$ (bottom left) and Res-1b (bottom right) regions at $\sqrt{s}=14 \mathrm{TeV}$. 
TABLE I. Event selection criteria in the mono- $W / Z$ search [31]. The symbols $j$ and $J$ are the small- $R$ and large- $R$ jets, respectively. $j_{i}$ 's are the small- $R$ jets ordered by their $p_{T}$ in decreasing order. Angles are defined in radians. See the text for details.

\begin{tabular}{|c|c|c|c|c|c|}
\hline & \multicolumn{4}{|c|}{ Merged topology } & Resolved topology \\
\hline$E_{T}^{\mathrm{miss}}$ & \multicolumn{4}{|c|}{$>250 \mathrm{GeV}$} & $>150 \mathrm{GeV}$ \\
\hline Jets, leptons & \multicolumn{4}{|c|}{$\geq 1 \mathrm{~J}, 0 \ell$} & $\geq 2 j, 0 \ell$ \\
\hline$b$ jets & \multicolumn{4}{|c|}{ No $b$-tagged jets outside of $J$} & $\leq 2 b$-tagged small- $R$ jets \\
\hline Multijet suppression & \multicolumn{5}{|c|}{$\begin{array}{c}\Delta \phi\left(\vec{E}_{T}^{\text {miss }}, J \text { or } j j\right)>2 \pi / 3 \\
\min _{i=1,2,3}\left[\Delta \phi\left(\vec{E}_{T}^{\text {miss }} j_{i}\right)\right]>\pi / 9 \\
\left|\vec{p}_{T}^{\text {miss }}\right|>30 \mathrm{GeV} \text { or } \geq 2 b \text { jets } \\
\Delta \phi\left(\vec{E}_{T}^{\text {miss }}, \vec{p}_{T}^{\text {miss }}\right)<\pi / 2\end{array}$} \\
\hline Signal properties & \multicolumn{5}{|r|}{$\begin{array}{c}p_{T}^{j_{1}}>45 \mathrm{GeV} \\
p_{T}^{j_{i}}>120(150) \mathrm{GeV} \text { for } 2(\geq 3) \text { jets }\end{array}$} \\
\hline Signal region & Ob-HP & Ob-LP & 1b-HP & 1b-LP & 1b-Res \\
\hline$J$ or $j j$ & $\mathrm{HP}$ & LP & HP & LP & $\Delta R_{j j}<1.4$ and $m_{j j} \in[65,105] \mathrm{GeV}$ \\
\hline$b$ jet & No $b$ jet & No $b$ jet & $1 b$ jet & $1 b$ jet & No $b$ jet \\
\hline
\end{tabular}

small. Therefore, the limit from the signal regions without $b$-tagged jets will give the most stringent limit.

The main SM backgrounds for these events originate from $t \bar{t}$ events and $Z+$ jets events where the $Z$ decays invisibly to neutrinos. The search found no excess on top of the backgrounds in $36.1 \mathrm{fb}^{-1}$ of data. Thus, we obtain the upper bounds on the signal events in each signal region from the experimental data and the expected number of SM backgrounds [31].

We set the $95 \%$ C.L. limit on the number of signal events $s$, such that

$$
\mathrm{CL}_{s}:=\frac{\sum_{k=0}^{n} \operatorname{Pois}(s+b \mid k)}{\sum_{k=0}^{n} \operatorname{Pois}(b \mid k)}=0.05, \quad \operatorname{Pois}(\lambda \mid k):=\frac{\lambda^{k} e^{-\lambda}}{k !},
$$

where $n$ and $b$ are the number of observed events and backgrounds, respectively. We also study the expected exclusion limit and discovery potential at the HL-LHC with an integrated luminosity of $3000 \mathrm{fb}^{-1}$. We simply assume that the number of backgrounds is scaled up by the integrated luminosity from the analysis with $36.1 \mathrm{fb}^{-1}$. The increase of the center-of-mass energy to $14 \mathrm{TeV}$, pile-up effects due to the high-luminosity environment, upgrades of the detector, and so on are neglected in this analysis. The signal events are generated with the same configuration as in the $13 \mathrm{TeV}$ analysis, but the center-of-mass energy is set to $14 \mathrm{TeV}$. The exclusion limit (discovery potential)

TABLE II. Upper limits on the number of signal events in the signal regions.

\begin{tabular}{lcccccc}
\hline \hline & 0b-HP & 0b-LP & 1b-HP & 1b-LP & 0b-Res & 1b-Res \\
\hline Current & 205.1 & 218.3 & 89.25 & 122.2 & 887.6 & 318.9 \\
HL-LHC $2 \sigma$ & 1777 & 2181 & 782.1 & 929.7 & 8235 & 2869 \\
HL-LHC $5 \sigma$ & 4442 & 5453 & 1955 & 2324 & 20587 & 7174 \\
\hline \hline
\end{tabular}

at the HL-LHC on the number of signal events $s$ is estimated as

$\frac{s}{\sqrt{b_{14 \mathrm{TeV}}}}=2(5), \quad b_{14 \mathrm{TeV}}=\frac{3000 \mathrm{fb}^{-1}}{36.1 \mathrm{fb}^{-1}} \times b_{13 \mathrm{TeV}}$,

where $b_{13 \mathrm{TeV}}$ is the number of backgrounds at $13 \mathrm{TeV}$ [31]. The upper limits of the signal events are summarized in Table II.

\section{Results}

Figure 8 shows the ratios of the number of events in the signal regions obtained by the simulation to the exclusion limits when the mass difference is $5 \mathrm{GeV}$. The solid lines are the current limit at $13 \mathrm{TeV}$, and the dashed lines are the $2 \sigma$ limit at $14 \mathrm{TeV}$ with $3 \mathrm{ab}^{-1}$ of data. The colors represent the signal regions. The mass region is excluded if any of the

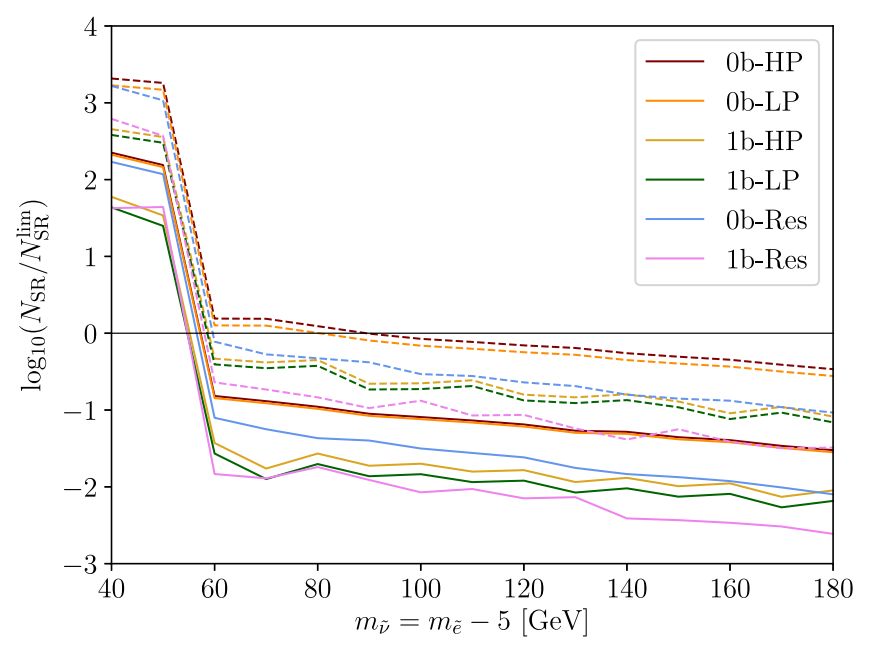

FIG. 8. Experimental limits to the mass-degenerate region. 


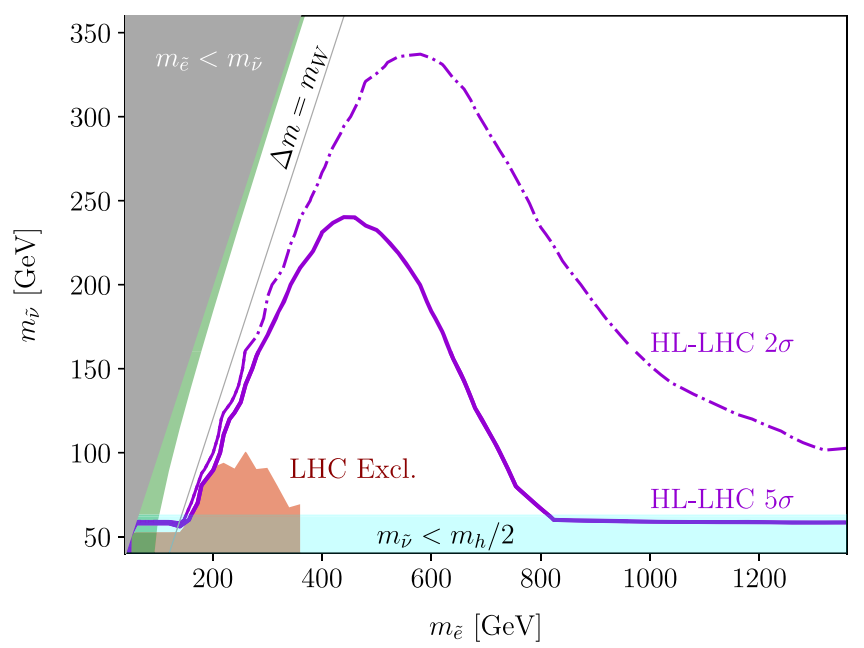

FIG. 9. Sneutrino-slepton parameter space, showing the constraints imposed by our analysis.

lines are above the black line. All of the slepton pair production channels (namely, $\tilde{\nu} \tilde{\nu}, \tilde{\nu} \tilde{e}, \tilde{e} \tilde{e}$ ) are included in the analysis, since the decay products of the charged sleptons are too soft to be reconstructed. We see that the Ob-HP signal region is the most sensitive to slepton pair production, and the 0b-LP signal region has a slightly weaker sensitivity. Although the efficiency of the 0b-Res topology is as large as that of the 0b-HP topology, the limit is weaker because of the large SM background. We see from Fig. 8 that the current limit on the sneutrino mass is about $55 \mathrm{GeV}$, and the expected limit from the full run of the HL-LHC is about $90 \mathrm{GeV}$. The HL-LHC exclusion limit may be sensitive to the off-shell production of sneutrino pairs, but the $5 \sigma$ discovery potential is not sensitive to that and the limit is about $60 \mathrm{GeV}$.

In Fig. 9 we see exclusions in the sneutrino-charged slepton mass plane. The gray region represents points where the charged slepton is lighter than the sneutrino and thus is not compatible with the sneutrino LSP scenario. The green region represents regions with mass splittings consistent with MSSM scenarios, with splittings given in Eq. (2). The sneutrinos are lighter than half of the Higgs boson mass in the cyan region, where they may be excluded by the Higgs invisible decay, unless the effective $A$ term $A_{\tilde{\nu}}$ is less than $\mathcal{O}(1) \mathrm{GeV}$. We have also placed a diagonal line to where $\Delta m=m_{W}$. In this figure, only the sneutrino pair production is included to obtain the limits at $\Delta m<m_{W}$. The productions involving a charged slepton may be irrelevant for the mono- $W / Z$ search because the decay products of charged sleptons would change the topology of signals, unless these are too soft to be detected. The limits when charged sleptons are invisible at the detector were already shown in Fig. 8. We expect that the limits would not change significantly for larger mass differences up to about $20 \mathrm{GeV}$. As the mass difference increases, the decays of charged sleptons will become visible. In particular, events with reconstructed leptons from off-shell $W$ bosons will be rejected by the requirement of the signal regions; see Table I. This will reduce the sensitivity of the mono- $W / Z$ search, although the effect will not be drastic due to the small phase space and branching fraction of $W$ bosons to leptons.

The mono- $W$ becomes very powerful for a mass difference larger than $m_{W}$. In this region, the production process is dominated by charged slepton-sneutrino pair production, where the charged slepton decays through an on-shell $W$ in the final state. For these events, we may exclude sneutrinos in the $80-100 \mathrm{GeV}$ range for charged sleptons between 200-300 GeV. However, once the charged slepton mass is sufficiently large, the production cross section drops, and the bound returns to $55 \mathrm{GeV}$, which is the bound for the production of sneutrino pairs alone. The purple curves show projections for the full $3 \mathrm{ab}^{-1}$ run of the HL-LHC. The solid line shows the $5 \sigma$ discovery potential in the mass plane. The dashed line gives a projection where $s / \sqrt{b_{14 \mathrm{TeV}}}=2$ and constitutes a $\sim 95 \%$ C.L. exclusion assuming no excess is seen. In the MSSM region, the projected bound for the HL-LHC is around $60 \mathrm{GeV}$ if the decay products of the charged slepton are visible, while it is around $90 \mathrm{GeV}$ if they are invisible, as shown in Fig. 8 .

\section{CONCLUSIONS}

The sneutrino (N)LSP scenario presents a challenge for discovering and constraining the slepton sector of supersymmetric models. We have used Higgs constraints and recast the ATLAS hadronic mono-boson search to bound slepton parameter space in light sneutrino scenarios. We see that $13 \mathrm{TeV}$ data can place absolute lower bounds on sneutrinos of around $60 \mathrm{GeV}$, an improvement over previous constraints from the LEP $Z$ invisible width. For more exotic regions of the sneutrino-charged slepton mass plane, we may place $80-100 \mathrm{GeV}$ bounds on sneutrinos for charged slepton masses in the $200-300 \mathrm{GeV}$ mass region. Projections for the HL-LHC may place a lower mass bound of around $90 \mathrm{GeV}$ for a MSSM sneutrino (N)LSP, assuming no excess is seen. Conversely, if the light sneutrino scenario exists we may place a $5 \sigma$ discovery potential of around $m_{\tilde{\nu}} \sim 60 \mathrm{GeV}$.

There are various further analyses that may be used in combination with our hadronic mono- $W / Z$ analysis to try to further improve the bounds and discovery potential of sleptons in the light sneutrino scenario. For example, a recasting of the monophoton analysis may also prove fruitful to zero in on this scenario. In addition, a new analysis of events with soft leptons and missing energy has proven useful for constraining Higgsinos [82]. Since we expect events that contain leptons of intermediate energy resulting from charged slepton decay into sneutrinos in slepton pair production processes, we may imagine new analyses that take advantage of lepton(s) plus missing energy signals. 


\section{ACKNOWLEDGMENTS}

The work of L. M. C., H. B. G., and J. K. is supported in part by the Department of Energy (DOE) under Award No. DESC0011726. The work of J. K. is supported in part by the Grant-in-Aid for Scientific Research from the Ministry of Education, Science, Sports and Culture (MEXT), Japan No. 18K13534.

[1] ATLAS Collaboration, Technical Report No. ATLASCONF-2020-002, CERN, Geneva, 2020.

[2] ATLAS Collaboration, Technical Report No. ATLASCONF-2019-040, CERN, Geneva, 2019.

[3] A. M. Sirunyan et al. (CMS Collaboration), Searches for physics beyond the standard model with the $M_{\mathrm{T} 2}$ variable in hadronic final states with and without disappearing tracks in proton-proton collisions at $\sqrt{s}=13 \mathrm{TeV}$, Eur. Phys. J. C 80, 3 (2020).

[4] A. M. Sirunyan et al. (CMS Collaboration), Search for supersymmetry in proton-proton collisions at $13 \mathrm{TeV}$ in final states with jets and missing transverse momentum, J. High Energy Phys. 10 (2019) 244.

[5] M. Aaboud et al. (ATLAS Collaboration), Search for longlived charginos based on a disappearing-track signature in pp collisions at $\sqrt{s}=13 \mathrm{TeV}$ with the ATLAS detector, J. High Energy Phys. 06 (2018) 022.

[6] J. Abdallah et al. (DELPHI Collaboration), Searches for supersymmetric particles in e+ e- collisions up to $208-\mathrm{GeV}$ and interpretation of the results within the MSSM, Eur. Phys. J. C 31, 421 (2003).

[7] M. Tanabashi et al. (Particle Data Group Collaboration), Review of particle physics, Phys. Rev. D 98, 030001 (2018).

[8] D. Decamp et al. (ALEPH Collaboration), Searches for new particles in $Z$ decays using the ALEPH detector, Phys. Rep. 216, 253 (1992).

[9] J. S. Hagelin, G. L. Kane, and S. Raby, Perhaps scalar neutrinos are the lightest supersymmetric partners, Nucl. Phys. B241, 638 (1984).

[10] L. E. Ibanez, The scalar neutrinos as the lightest supersymmetric particles and cosmology, Phys. Lett. 137B, 160 (1984).

[11] P. Meade, N. Seiberg, and D. Shih, General gauge mediation, Prog. Theor. Phys. Suppl. 177, 143 (2009).

[12] A. Rajaraman, Y. Shirman, J. Smidt, and F. Yu, Parameter space of general gauge mediation, Phys. Lett. B 678, 367 (2009).

[13] L. M. Carpenter, Surveying the phenomenology of general gauge mediation, arXiv:0812.2051.

[14] P. Fayet, Massive gluinos, Phys. Lett. 78B, 417 (1978).

[15] J. Polchinski and L. Susskind, Breaking of supersymmetry at intermediate-energy, Phys. Rev. D 26, 3661 (1982).

[16] L. Hall and L. Randall, U(1)-R symmetric supersymmetry, Nucl. Phys. B352, 289 (1991).

[17] L. M. Carpenter, Exploring non-universal gauge mediation, arXiv:1712.10269.

[18] L. M. Carpenter, Antisplit supersymmetry, J. High Energy Phys. 10 (2017) 205.
[19] M. Bernhardt, S. Das, H. Dreiner, and S. Grab, Sneutrino as lightest supersymmetric particle in $B_{3}$ mSUGRA models and signals at the LHC, Phys. Rev. D 79, 035003 (2009).

[20] V. Berezinsky, A. Bottino, J. R. Ellis, N. Fornengo, G. Mignola, and S. Scopel, Neutralino dark matter in supersymmetric models with nonuniversal scalar mass terms, Astropart. Phys. 5, 1 (1996).

[21] M. Drees, M. M. Nojiri, D. Roy, and Y. Yamada, Light Higgsino dark matter, Phys. Rev. D 56, 276 (1997); Erratum, Phys. Rev. D 64, 039901 (2001).

[22] P. Nath and R. L. Arnowitt, Nonuniversal soft SUSY breaking and dark matter, Phys. Rev. D 56, 2820 (1997).

[23] A. Katz and B. Tweedie, Signals of a Sneutrino (N)LSP at the LHC, Phys. Rev. D 81, 035012 (2010).

[24] G. Aad et al. (ATLAS Collaboration), Search for electroweak production of charginos and sleptons decaying into final states with two leptons and missing transverse momentum in $\sqrt{s}=13 \mathrm{TeV} p p$ collisions using the ATLAS detector, Eur. Phys. J. C 80, 123 (2020).

[25] A. M. Sirunyan et al. (CMS Collaboration), Search for supersymmetric partners of electrons and muons in protonproton collisions at $\sqrt{s}=13 \mathrm{TeV}$, Phys. Lett. B 790, 140 (2019).

[26] A. Anandakrishnan, L. M. Carpenter, and S. Raby, Degenerate gaugino mass region and mono-boson collider signatures, Phys. Rev. D 90, 055004 (2014).

[27] H. Baer, A. Mustafayev, and X. Tata, Monojets and monophotons from light Higgsino pair production at LHC14, Phys. Rev. D 89, 055007 (2014).

[28] G. F. Giudice, T. Han, K. Wang, and L.-T. Wang, Nearly degenerate gauginos and dark matter at the LHC, Phys. Rev. D 81, 115011 (2010).

[29] J. Goodman, M. Ibe, A. Rajaraman, W. Shepherd, T. M. P. Tait, and H.-B. Yu, Constraints on dark matter from colliders, Phys. Rev. D 82, 116010 (2010).

[30] D. Abercrombie et al., Dark matter benchmark models for early LHC run-2 searches: Report of the ATLAS/CMS dark matter forum, Phys. Dark Universe 27, 100371 (2020).

[31] M. Aaboud et al. (ATLAS Collaboration), Search for dark matter in events with a hadronically decaying vector boson and missing transverse momentum in $p p$ collisions at $\sqrt{s}=$ $13 \mathrm{TeV}$ with the ATLAS detector, J. High Energy Phys. 10 (2018) 180.

[32] G. Bennett et al. (Muon g-2 Collaboration), Final Report of the Muon E821 anomalous magnetic moment measurement at BNL, Phys. Rev. D 73, 072003 (2006).

[33] C. Arina and N. Fornengo, Sneutrino cold dark matter, a new analysis: Relic abundance and detection rates, J. High Energy Phys. 11 (2007) 029. 
[34] M. A. Bernhardt, S. Prasad Das, H. K. Dreiner, and S. Grab, Sneutrino LSPs in R-parity violating minimal supergravity models, AIP Conf. Proc. 1078, 306 (2009).

[35] J. L. Lopez, D. V. Nanopoulos, and X. Wang, Large (g-2)mu in $\mathrm{SU}(5) \times \mathrm{U}(1)$ supergravity models, Phys. Rev. D 49, 366 (1994).

[36] U. Chattopadhyay and P. Nath, Probing supergravity grand unification in the Brookhaven g-2 experiment, Phys. Rev. D 53, 1648 (1996).

[37] T. Moroi, The Muon anomalous magnetic dipole moment in the minimal supersymmetric standard model, Phys. Rev. D 53, 6565 (1996); Erratum, Phys. Rev. D 56, 4424 (1997).

[38] M. Endo, K. Hamaguchi, S. Iwamoto, and K. Yanagi, Probing minimal SUSY scenarios in the light of muon $g-2$ and dark matter, J. High Energy Phys. 06 (2017) 031.

[39] M. W. Goodman and E. Witten, Detectability of certain dark matter candidates, Phys. Rev. D 31, 3059 (1985).

[40] K. Freese, Can scalar neutrinos or massive dirac neutrinos be the missing mass?, Phys. Lett. 167B, 295 (1986).

[41] T. Falk, K. A. Olive, and M. Srednicki, Heavy sneutrinos as dark matter, Phys. Lett. B 339, 248 (1994).

[42] L. J. Hall, T. Moroi, and H. Murayama, Sneutrino cold dark matter with lepton number violation, Phys. Lett. B 424, 305 (1998).

[43] M. Cirelli, N. Fornengo, and A. Strumia, Minimal dark matter, Nucl. Phys. B753, 178 (2006).

[44] D. Tucker-Smith and N. Weiner, The status of inelastic dark matter, Phys. Rev. D 72, 063509 (2005).

[45] T. Moroi, H. Murayama, and M. Yamaguchi, Cosmological constraints on the light stable gravitino, Phys. Lett. B 303, 289 (1993).

[46] J. L. Feng, A. Rajaraman, and F. Takayama, Superweakly Interacting Massive Particles, Phys. Rev. Lett. 91, 011302 (2003).

[47] J. L. Feng, A. Rajaraman, and F. Takayama, SuperWIMP dark matter signals from the early universe, Phys. Rev. D 68 , 063504 (2003).

[48] J. R. Ellis, K. A. Olive, Y. Santoso, and V. C. Spanos, Gravitino dark matter in the CMSSM, Phys. Lett. B 588, 7 (2004).

[49] J. L. Feng, S.-f. Su, and F. Takayama, SuperWIMP gravitino dark matter from slepton and sneutrino decays, Phys. Rev. D 70, 063514 (2004).

[50] T. Goto and M. Yamaguchi, Is axino dark matter possible in supergravity?, Phys. Lett. B 276, 103 (1992).

[51] E. Chun, J. E. Kim, and H. P. Nilles, Axino mass, Phys. Lett. B 287, 123 (1992).

[52] L. Covi, H.-B. Kim, J. E. Kim, and L. Roszkowski, Axinos as dark matter, J. High Energy Phys. 05 (2001) 033.

[53] L. Covi, L. Roszkowski, R. Ruiz de Austri, and M. Small, Axino dark matter and the CMSSM, J. High Energy Phys. 06 (2004) 003.

[54] K.-Y. Choi, L. Covi, J. E. Kim, and L. Roszkowski, Axino cold dark matter revisited, J. High Energy Phys. 04 (2012) 106.

[55] N. G. Deshpande and E. Ma, Pattern of symmetry breaking with two Higgs doublets, Phys. Rev. D 18, 2574 (1978).

[56] P. Agrawal, Z. Chacko, and C. B. Verhaaren, Leptophilic dark matter and the anomalous magnetic moment of the muon, J. High Energy Phys. 08 (2014) 147.
[57] S. Baek, Dark matter, and muon $(g-2)$ in local $U(1)_{L_{\mu}-L_{\tau}}{ }^{-}$ extended Ma model, Phys. Lett. B 756, 1 (2016).

[58] K. Kowalska and E. M. Sessolo, Expectations for the muon g-2 in simplified models with dark matter, J. High Energy Phys. 09 (2017) 112.

[59] L. Calibbi, R. Ziegler, and J. Zupan, Minimal models for dark matter and the muon $g-2$ anomaly, J. High Energy Phys. 07 (2018) 046.

[60] J. Kawamura, S. Okawa, and Y. Omura, Current status and muon $g-2$ explanation of lepton portal dark matter, J. High Energy Phys. 08 (2020) 042.

[61] P. J. Fox, A. E. Nelson, and N. Weiner, Dirac gaugino masses and supersoft supersymmetry breaking, J. High Energy Phys. 08 (2002) 035.

[62] J. Schechter and J. W. F. Valle, Neutrino masses in $\mathrm{SU}(2) \times$ U(1) theories, Phys. Rev. D 22, 2227 (1980).

[63] G. Lazarides, Q. Shafi, and C. Wetterich, Proton lifetime and fermion masses in an SO(10) model, Nucl. Phys. B181, 287 (1981).

[64] R. N. Mohapatra and G. Senjanovic, Neutrino masses and mixings in gauge models with spontaneous parity violation, Phys. Rev. D 23, 165 (1981).

[65] C. Wetterich, Neutrino masses and the scale of B-L violation, Nucl. Phys. B187, 343 (1981).

[66] R. N. Mohapatra, Mechanism for Understanding Small Neutrino Mass in Superstring Theories, Phys. Rev. Lett. 56, 561 (1986).

[67] R. N. Mohapatra and J. W. F. Valle, Neutrino mass and baryon number nonconservation in superstring models, Phys. Rev. D 34, 1642 (1986).

[68] ATLAS Collaboration, Search for invisible Higgs boson decays with vector boson fusion signatures with the ATLAS detector using an integrated luminosity of $139 \mathrm{fb}^{-1}$.

[69] E. Bagnaschi, G. F. Giudice, P. Slavich, and A. Strumia, Higgs mass and unnatural supersymmetry, J. High Energy Phys. 09 (2014) 092.

[70] G. Bozzi, B. Fuks, and M. Klasen, Threshold resummation for slepton-pair production at hadron colliders, Nucl. Phys. B777, 157 (2007).

[71] B. Fuks, M. Klasen, D. R. Lamprea, and M. Rothering, Precision predictions for electroweak superpartner production at hadron colliders with Resummino, Eur. Phys. J. C 73, 2480 (2013).

[72] B. Fuks, M. Klasen, D. R. Lamprea, and M. Rothering, Revisiting slepton pair production at the Large Hadron Collider, J. High Energy Phys. 01 (2014) 168.

[73] J. Fiaschi and M. Klasen, Neutralino-chargino pair production at NLO + NLL with resummation-improved parton density functions for LHC Run II, Phys. Rev. D 98, 055014 (2018).

[74] W. Beenakker, M. Klasen, M. Kramer, T. Plehn, M. Spira, and P. M. Zerwas, The Production of Charginos/Neutralinos and Sleptons at Hadron Colliders, Phys. Rev. Lett. 83, 3780 (1999).

[75] B. Fuks, M. Klasen, D. R. Lamprea, and M. Rothering, Gaugino production in proton-proton collisions at a centerof-mass energy of $8 \mathrm{TeV}$, J. High Energy Phys. 10 (2012) 081.

[76] J. Alwall, R. Frederix, S. Frixione, V. Hirschi, F. Maltoni, O. Mattelaer, H. S. Shao, T. Stelzer, P. Torrielli, and M. Zaro, 
The automated computation of tree-level and next-to-leading order differential cross sections, and their matching to parton shower simulations, J. High Energy Phys. 07 (2014) 079.

[77] T. Sjostrand, S. Mrenna, and P.Z. Skands, A brief introduction to PYTHIA 8.1, Comput. Phys. Commun. 178, 852 (2008).

[78] J. de Favereau, C. Delaere, P. Demin, A. Giammanco, V. Lemaître, A. Mertens, and M. Selvaggi (DELPHES 3 Collaboration), DELPHES 3, A modular framework for fast simulation of a generic collider experiment, J. High Energy Phys. 02 (2014) 057.
[79] M. Cacciari, G. P. Salam, and G. Soyez, The anti- $k_{t}$ jet clustering algorithm, J. High Energy Phys. 04 (2008) 063.

[80] M. Cacciari, G. P. Salam, and G. Soyez, FastJet user manual, Eur. Phys. J. C 72, 1896 (2012).

[81] D. Krohn, J. Thaler, and L.-T. Wang, Jet trimming, J. High Energy Phys. 02 (2010) 084.

[82] G. Aad et al. (ATLAS Collaboration), Searches for electroweak production of supersymmetric particles with compressed mass spectra in $\sqrt{s}=13 \mathrm{TeV} p p$ collisions with the ATLAS detector, Phys. Rev. D 101, 052005 (2020). 